\title{
Editorial: innovative quantum materials
}

\author{
Vittorio Cataudella ${ }^{\text {a }}$ Procolo Lucignano, Carmine Antonio Perroni
}

Dipartimento di Fisica "Ettore Pancini” and CNR-SPIN, Università degli Studi di Napoli "Federico II", c/o Complesso di Monte S. Angelo, via Cinthia, 80126 Napoli, Italy

(C) The Author(s), under exclusive licence to Società Italiana di Fisica and Springer-Verlag GmbH Germany, part of Springer Nature 2021

\begin{abstract}
Quantum Materials are materials where the manifestation of the quantum mechanical nature of matter constituents, which comes into evidence at the macroscopic scale, is used to obtain new functionalities. The study of quantum materials is relevant both on the fundamental and on the applied side. Indeed, this class of materials provides a common thread between physics, materials science and engineering. The focus is on emergent excitations, such as Dirac and Majorana fermions. In particular, it analyzes their sensitivity to external perturbations, such as electric and magnetic fields, and boundary conditions that can be controlled by surface/edge terminations, defect states and nanostructuring. The topical issue provides a broad description of innovative quantum materials discussing a variety of different phenomena: (1) interference phenomena in quantum devices made up of a topological insulator, (2) bound states in finite length nanowires with an inhomogeneous spin-orbit coupling profile relevant for Majorana physics, (3) sensitivity of graphene transport properties to defect states and edge functionalization, (4) role of Moiré phonons on the energy properties of twisted bilayer graphene at the magic angle important for van der Waals materials, (5) emergent spin excitations and anisotropic magnetotransport properties in iridates, (6) magnetoelectric couplings and improper magnetoelectric behavior in manganites significant for the realization of novel spintronic devices.
\end{abstract}

During the last decades, the possibility to engineer materials at the nanoscale paved the way to a new era in condensed matter physics in which innovative materials can provide new functionalities. In this context an important role is played by the so-called quantum materials [1], where the manifestation of the quantum mechanical nature of matter constituents comes into evidence at the macroscopic scale. A common thread in the field of quantum materials is the concept of emergent phenomena [2] whose study is no longer restricted to strongly correlated low-dimensional systems. The focus is on emergent excitations, such as Dirac and Majorana fermions, analyzing their sensitivity to external perturbations, like electric and magnetic fields, and boundary conditions, due to surface/edge terminations, defect states and nanostructuring. The aim of the topical issue is to provide a broad description of quantum materials discussing a variety of different phenomena.

An important class of new quantum materials is given by topological insulators [3] which are characterized in terms of topological invariants, reflecting the geometric properties of the single-electron wave functions. The interest on these materials comes mainly from the

\footnotetext{
a e-mail: Vittorio.Cataudella@unina.it (corresponding author)
} 
presence of robust surface metallic states with exceptional properties, such as dissipationless charge transport. In this focus point, interference phenomena in quantum devices made up of a topological insulator are described in Ref [4], showing that the inclusion of the spin-degreeof-freedom leads to a modification of the diffraction patterns that depend on the geometric parameters of the system.

Other relevant examples of topologically protected states of matter are given by Weyl fermions in topological semimetals [5] and Majorana fermions in topological superconductors [6], which are relevant for the burgeoning field of topological quantum computation [7]. Relevant for Majorana physics are spin-orbit coupled nanowires. Within this focus point, finite length nanowires with an inhomogeneous spin-orbit coupling profile are described in Ref [8], showing that in general two types of bound states arise in the nanowire, namely confinement bound states and interface bound states, which, under an applied magnetic field, can transform from the one in the other determining a redistribution of the electron charge.

Another important class of quantum materials includes two-dimensional materials, such as graphene [8] and transition metal dichalcogenides [9]. In graphene, one of the most interesting phenomena is the strong sensitivity of transport properties to external perturbations.

In this focus point, graphene defect states and edge functionalization of graphene nanoribbons are studied in Refs. [10, 11], respectively. In particular, in Ref. [10], the existence of localized states in close vicinity of a linear defect is demonstrated, showing that these states have insulating or conducting character. Moreover, in Ref [11] of this issue, an even-odd conductance effect induced by edge functionalization with aromatic molecules is theoretically analyzed in graphene nanoribbons pointing out a subtle interplay between the electronic states of the guest molecule that are spatially localized on the binding sites and those of the host nanoribbon. Not only transport but also optoelectronic properties are investigated in van der Waals materials with the aim to realize innovative devices. Recently, twistronics $[12,13]$ is exploiting the manipulation of a new degree of freedom that is the twist angle between two and more layers of two-dimensional van der Waals heterostructures, to construct artificial materials with new fascinating properties. In Ref [14] of this issue, Moirè phonons are included in a continuum formalism to discuss their relevance on the energy properties of twisted bilayer graphene at the magic angle.

Finally, a wide variety of emergent phenomena can occur at the surfaces or interfaces of oxide materials, among which manganites and iridates, where effects due to spin-orbit interaction are not negligible [15]. Indeed, in addition to strong electron correlations and low dimensionality, energetic terms due to relativistic corrections contribute to stabilize emergent excitations. In Ref [16] of this focus point, resonant inelastic X-ray scattering measurements on three-dimensional bulk $\mathrm{CaIrO}_{3}$ reveal a prototypical pseudospinon continuum, a hallmark of one-dimensional magnetic systems, which is attributed to the fundamental difference in the magnetic interactions between the pseudospins along the corner- and edge-sharing bonds. Reference [17] of this focus point analyzes the anisotropic magnetoresistance of $\mathrm{SrIrO}_{3}$ thin films revealing the appearance of a fourfold symmetric component above a critical magnetic field. Finally, Ref [18] studies the magnetoelectric coupling in epitaxial $\mathrm{BiMnO}_{3}$ thin films which exhibit an improper magnetoelectric behavior opening perspectives for the realization of novel spintronic devices.

\section{References}

1. B. Keimer, J.E. Moore, Nat. Phys. 13, 1045 (2017)

2. X.G. Wen, Quantum Field Theory of Many-Body Systems (Oxford University Press, Oxford, 2004) 
3. M.Z. Hasan, C.L. Kane, Rev. Mod. Phys. 82, 3045 (2010)

4. D. Bercioux, T.L. van den Berg, D. Ferraro, J. Rech, T. Jonckheere, T. Martin, Eur. Phys. J. Plus 8, 811 (2020)

5. N.P. Armitage, E.J. Mele, A. Vishwanath, Mod. Phys. 90, 015001 (2018)

6. R.M. Lutchyn, E.P.A.M. Bakkers, L.P. Kouwenhoven, P. Krogstrup, C.M. Marcus, Y. Oreg, Nat. Rev. Mater. 3, 52 (2018)

7. C. Nayak, S.H. Simon, A. Stern, M. Freedman, S. Das Sarma, Rev. Mod. Phys. 80, 1083 (2008)

8. L. Rossi, F. Dolcini, F. Rossi, Eur. Phys. J. Plus 8, 587 (2020)

9. S. Manzeli, D. Ovchinnikov, D. Pasquier, O.V. Yazyev, A. Kis, Nat. Rev. Mater. 2, 17033 (2017)

10. F. Romeo, Eur. Phys. J. Plus 8, 446 (2020)

11. K. Cernevics, M. Pizzochero, O.V. Yazyev, Eur. Phys. J. Plus 8, 681 (2020)

12. S. Carr, S. Fang, E. Kaxiras, Nat. Rev. Mater. 5, 748 (2020)

13. P. Lucignano, D. Alfe, V. Cataudella, D. Ninno, G. Cantele, Phys. Rev. B 99, 195419 (2019)

14. M. Angeli, M. Fabrizio, Eur. Phys. J. Plus 8, 630 (2020)

15. H.Y. Hwang, Y. Iwasa, M. Kawasaki, B. Keimer, N. Nagaosa, Y. Tokura, Nat. Mater. 11, 103-113 (2012)

16. M. Rossi et al., Eur. Phys. J. Plus 8, $676(2020)$

17. D.J. Groenendijk et al., Eur. Phys. J. Plus 8, 627 (2020)

18. G.M. De Luca et al., Eur. Phys. J. Plus 8, 473 (2020) 